\title{
Original
}

\section{c-MAF deletion in adult C57BL/6J mice induces cataract formation and abnormal differentiation of lens fiber cells}

\author{
Mitsunori FUJINO ${ }^{1,2)}$, Asuka TAGAMI ${ }^{1)}$, Masami OJIMA ${ }^{3)}$, Seiya MIZUNO ${ }^{3)}$, \\ Ahmed M. ABDELLATIF ${ }^{7)}$, Akihiro KUNO ${ }^{1)}$ and Satoru TAKAHASHI ${ }^{1,3-6)}$ \\ 1) Department of Anatomy and Embryology, Faculty of Medicine, University of Tsukuba, 1-1-1 Tennodai, Tsukuba, Ibaraki \\ 305-8575, Japan \\ 2) Ph.D. Program in Human Biology, School of Integrative and Global Majors, University of Tsukuba, 1-1-1 Tennodai, \\ Tsukuba, Ibaraki 305-8575, Japan \\ 3) Laboratory Animal Resource Center (LARC), University of Tsukuba, 1-1-1 Tennodai, Tsukuba, Ibaraki 305-8575, Japan \\ ${ }^{4)}$ Life Science Center, Tsukuba Advanced Research Alliance (TARA), University of Tsukuba, 1-1-1 Tennodai, Tsukuba, Ibaraki \\ 305-8575, Japan \\ 5) International Institute for Integrative Sleep Medicine (WPI-IIIS), University of Tsukuba, 1-1-1 Tennodai, Tsukuba, Ibaraki \\ 305-8575, Japan \\ 6) Transborder Medical Research Center, Faculty of Medicine, University of Tsukuba, 1-1-1 Tennodai, Tsukuba, Ibaraki \\ 305-8575, Japan \\ 7) Department of Anatomy and Embryology, Faculty of Veterinary Medicine, Mansoura University, Mansoura 35516, Egypt
}

\begin{abstract}
The transcription factor c-MAF is a member of the large MAF family, members of which possess transactivation and bZIP domains. c-MAF plays an important role in lens formation, T-lymphocyte differentiation, hypertrophic chondrocyte differentiation, and kidney development in mouse embryos. However, because homozygous deletion of $c-M a f$ in C57BL/6J mice causes embryonic lethality, the functions of c-MAF in adult mice remain largely uninvestigated. To address this issue, we generated $c-M a f$ floxed $(c-M a f f / f f) C 57 \mathrm{BL} / 6 \mathrm{~J}$ mice and established tamoxifen-inducible $c-M a f$ knockout mice (c-Mafl/ff; CAG-Cre-ER ${ }^{T M}$ mice, $c-M a f^{\Delta T A M}$ ). After tamoxifen injection, adult $c-M a f^{\triangle T A M}$ mice showed successful deletion of $\mathrm{c}$-Maf protein and developed severe cataracts; cataracts are also seen in human patients who have mutations in the $c-M A F$ DNA binding domain. Furthermore, adult $c-M a f^{\triangle T A M}$ mice exhibited abnormal lens structure and impaired differentiation of lens fiber cells. In summary, we established $c-M a f^{f / f f}$ and $c-M a f^{\triangle T A M}$ C57BL/6J mice, which can be useful animal models for the investigation of c-MAF function in various developmental stages and can also be used as a disease model for cataracts.
\end{abstract}

Key words: cataract, c-MAF, lens epithelial cell, lens fiber cell

\section{Introduction}

The transcription factor c-MAF belongs to the large MAF family, all members of which have a basic region and a leucine zipper domain. The leucine zipper domain mediates dimer formation, and the basic region allows the protein to bind DNA to Maf recognition elements (MARE). In addition to these domains, MAF family members are characterized by having an acidic transactivation domain in their N-terminal region [1].

Previous studies have reported that mutations in the DNA binding and transactivation domains of human $c$ $M A F$ are associated with familial juvenile cataracts [4, 9, 18, 26]. Moreover, $c$-Maf knockout embryos showed impaired lens fiber cell differentiation and lens formation due to dysregulation of Crystallin gene expression dur-

(Received 25 October 2019 / Accepted 24 December 2019 / Published online in J-STAGE 21 January 2020)

Corresponding authors: A. Kuno.e-mail: akuno@md.tsukuba.ac.jp

S.Takahashi.e-mail: satoruta@md.tsukuba.ac.jp

Supplementary Table: refer to J-STAGE: https://www.jstage.jst.go.jp/browse/expanim

c) $\$$ This is an open-access article distributed under the terms of the Creative Commons Attribution Non-Commercial No Derivatives cC) (by-nc-nd) License <http://creativecommons.org/licenses/by-nc-nd/4.0/>

(C)2020 Japanese Association for Laboratory Animal Science 
ing the differentiation of primary posterior lens fibers $[10,11]$. These results indicate that c-MAF is important for lens maintenance. However, the postnatal functions of c-MAF have remained largely unknown because $c$ Maf null mice on a C57BL/6J background exhibit embryonic lethality due to impaired definitive erythropoiesis in fetal liver cells [13].

The lens is composed of lens epithelial cells at the anterior pole and lens fiber cells at the posterior pole. Lens epithelial cells differentiate into lens fiber cells in a transitional region that is known as a germinative zone; lens fiber cells migrate toward the core of lenses and form lens nuclear cells. Crystallins are soluble proteins found in lenses, and there are three types: $\alpha, \beta$ and $\gamma$-Crystallin. $\alpha$-Crystallin is distributed in lens epithelial cells and lens fiber cells; in contrast, $\beta$ - and $\gamma$-Crystallin are only found in lens fiber cells. Crystallins are involved in lens development and maintenance, and they control lens cell proliferation and differentiation [3, 22, 24]. Moreover, c-MAF is one of the key transcription factors that regulates Crystallin expression $[10,11,21]$.

Herein, for the purpose of investigating c-MAF functions in adults, we generated $c$-Mafflff mice on a C57BL6/J background and tamoxifen-inducible $c-M a f$ conditional knockout mice (c-Maftlfl and CAG-Cre-ER $R^{T M}$ offspring were named $c$-Maf ${ }^{\triangle T A M}$ ) using CRISPR/Cas9 technology. Our analysis revealed that the $c-M a f^{\triangle T A M}$ mice had disrupted lens structures, abnormal differentiation of epithelial cells and cataracts, which recapitulate the human phenotype of $c-M A F$ mutations. These data reveal that these model mice can be used for investigating c-MAF function in adults.

\section{Materials and Methods}

\section{Animals}

Mice were kept under specific pathogen-free conditions in the Laboratory Animal Resource Center of the University of Tsukuba. All experiments in this study were performed in compliance with the guidelines of the Care and Use of Laboratory Animal Resource Center at the University of Tsukuba, and they were approved by the Institutional Review Board of the university. Male and female $\mathrm{C} 57 \mathrm{BL} / 6 \mathrm{~J}$ mice ( 8 weeks old) were purchased from SLC Japan (Tokyo, Japan). Mice with the floxed allele (c-Mafflff) were bred with Ayul-Cre mice [20] $\left(c-M a f^{+/ f} ; \mathrm{Ayu} 1-\mathrm{Cre}\right)$ to generate mice lacking the c-Maf gene (c-Mafflf; ; Ayu1-Cre). CAG-Cre-ER ${ }^{T M}$ mice [5] were used to generate $c-M a f^{\triangle T A M}$ mice. $C A G$-Cre$E R^{T M}$ transgenic mice were purchased from the Jackson Laboratory (Bar Harbor, Maine).

To activate the Cre recombination system, 8-week-old
$c-M a f^{\triangle T A M}$ and control mice were injected intraperitoneally with $75 \mathrm{mg} / \mathrm{kg}$ tamoxifen for 5 consecutive days. Tamoxifen (TAM, Sigma-Aldrich, T5648) was dissolved in ethanol and then mixed with corn oil as described previously [16]. Four mice from each group were sacrificed 7 months after TAM injection for histopathological and qPCR analyses of the lens.

\section{Genotyping of mice}

Genomic DNA was isolated from mouse tails, and PCR was performed to identify the floxed allele, Ayu1$\mathrm{Cre}$ and the $c-M a f$ deletion; the primers used are listed in Supplementary Table 1A. The wild-type PCR product was 431 bp in length, the floxed PCR product was 493 bp in length, the Ayul-Cre PCR product was $269 \mathrm{bp}$ in length, and the $c-M a f$ deletion PCR product was $993 \mathrm{bp}$ in length.

\section{Histopathological and immunohistochemical analyses}

For IHC staining, lenses were extracted from $c-\mathrm{Maf}^{+/+}$, $c-M a f^{f l f l}$ and $c-M a f^{f l f f}$; Ayul-Cre embryos at E14.5 and were fixed in $4 \%$ PFA at $4{ }^{\circ} \mathrm{C}$ for $24 \mathrm{~h}$; then, The lenses were embedded in paraffin, and $5 \mu \mathrm{m}$ sections were cut and placed on slides. The paraffin sections were subjected to paraffin removal and then were washed with PBS and distilled water. Then, these sections were permeabilized with $0.3 \%$ Triton X-100/PBS before undergoing antigen retrieval for $10 \mathrm{~min}$ at $121^{\circ} \mathrm{C}$, which was followed by incubation on ice for $1 \mathrm{~h}$ and blocking for $1 \mathrm{~h}$ in PBS with 5\% BSA and 10\% donkey serum. Then, these sections were incubated overnight at $4{ }^{\circ} \mathrm{C}$ with the following primary antibodies: rabbit anti-c-Maf (1:200; A300-613A; Bethyl, Montgomery, TX, USA) and mouse anti-E-cadherin $(1: 200 ; 610181$; BD Transduction Laboratories, Franklin Lakes, NJ, USA). Antigens were visualized using a secondary antibody conjugated to Alexa Fluor 594 (1:500; Life Technologies, Carlsbad, CA, USA) for c-Maf and Alexa Fluor 488 for E-cadherin; the nuclei were labeled with Hoechst 33342 (Molecular Probes). The sections were mounted with Fluoromount (Diagnostic BioSystems, Pleasanton, CA, USA). All images were captured with a fluorescence microscope (Biorevo BZ-9000; Keyence, Tokyo, Japan).

\section{Quantitative (q)PCR analysis}

Total RNA was extracted from the eye of $c-M a f^{\triangle T A M}$ and $c$-Mafflfl mice using ISOGEN Reagent (NIPPON GENE, Tokyo, Japan) 7 months after TAM injection. cDNA was synthesized in accordance with the protocol of a QuantiTect reverse transcription kit (Qiagen, Hilden, Germany). qPCR was performed in duplicate with a 
Thermal Cycler Dice real-time system (Takara, Shiga, Japan) using THUNDERBIRD SYBR qPCR Mix (Toyobo, Tokyo, Japan). The primers that were used for amplification and detection are listed in Supplementary Table 1B. The expression of target genes was normalized to the expression of Hprt. Each sample was tested in duplicate.

\section{Statistical analysis}

All data were presented as the mean and the standard error of the mean (SEM). To assess whether differences between $c$-Maf ${ }^{\triangle T A M}$ and control mice were statistically significant, a minimum of three biological replicates were analyzed using Welch's $t$ test, and a $P$-value $<0.05$ was considered significant.

\section{Results}

\section{Generation of c-Maf $\triangle$ TAM mice}

We applied CRISPR/Cas9 methods to generate double-stranded DNA with a floxed $c-M a f$ allele (Fig. 1A). The targeting vector contained a c-MAF protein-coding sequence and two loxP sites. To confirm the insertion of the floxed allele $\left(c-M a f^{f l f f}\right)$ and the Cre-dependent excision of the floxed allele, $c$-Maf ${ }^{\text {flf } f}$; Ayul-Cre mice were generated by mating $c-M a f^{f l / f}$ and $c-M a f^{+/ f}$; Ayul-Cre mice [20]. The loxP insertion at the $c$-Maflocus, the Cre insertion in the Ayul-Cre mice and the c-Maf deletion were all verified by genotyping PCR (Fig. 1B).

To confirm the $c$-Maf deletion at the protein and phenotype levels, we investigated the lenses of $c$-Mafflff; Ayu1-Cre mice because a previous study reported that c-MAF is expressed in lens fiber cells and that $c-M a f$

A

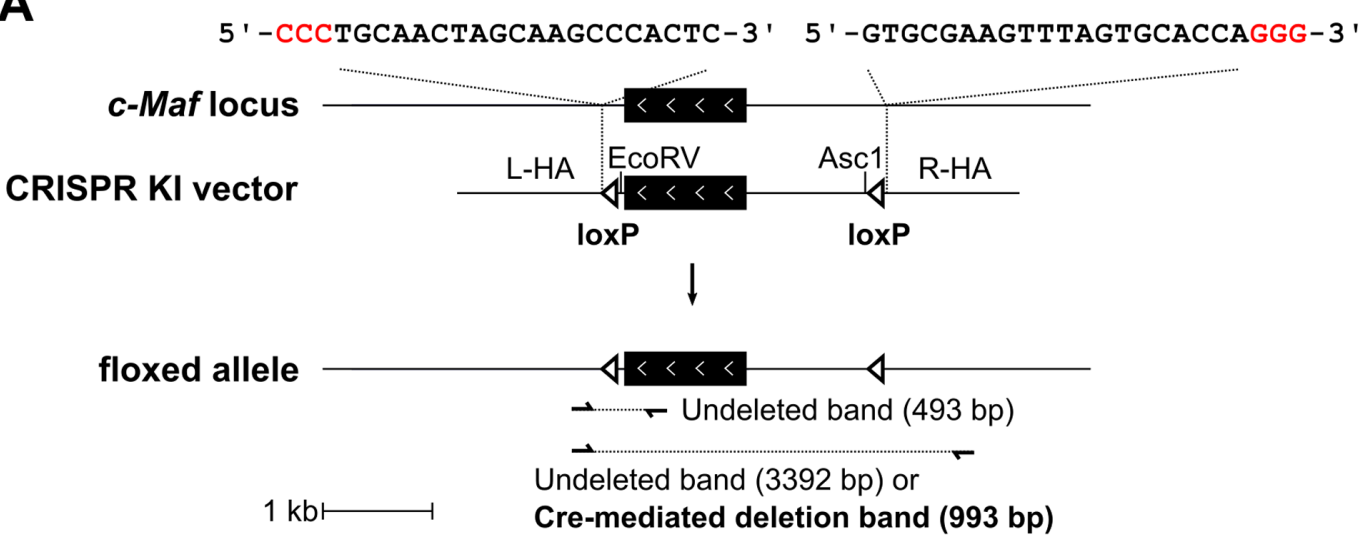

B

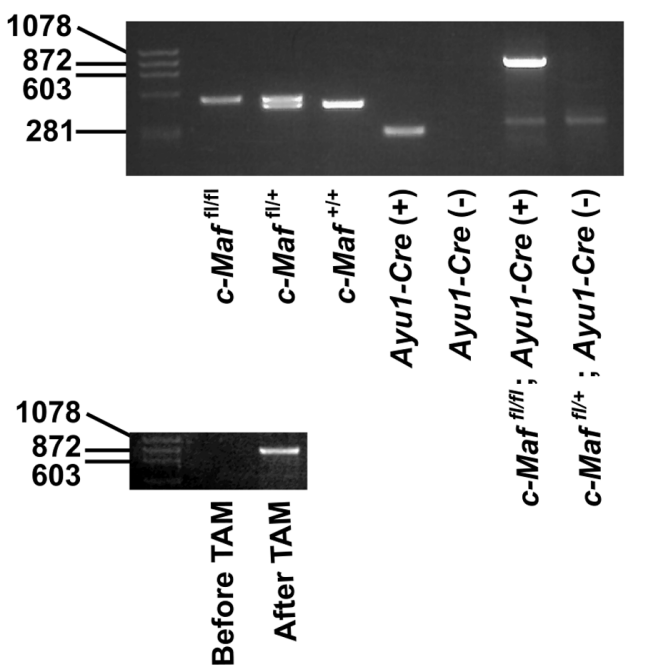

C
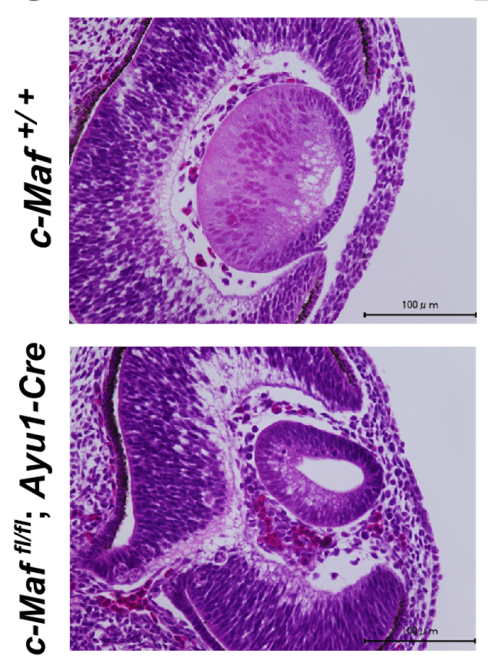

D
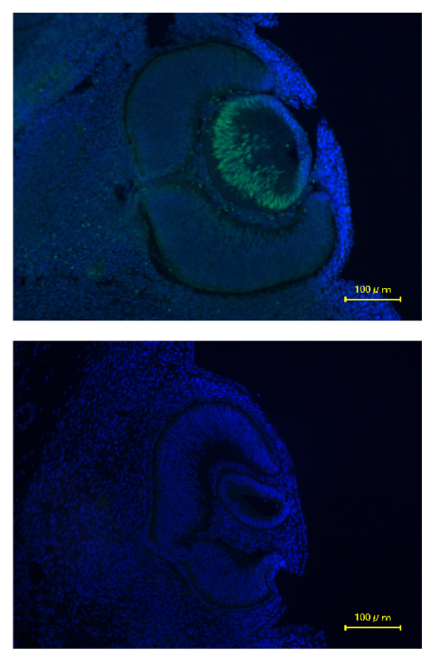

Fig. 1. Generation of $c$-Maf conditional knockout mice. (A) Schematic targeting strategy for the generation of floxed alleles in the $c$-Maf locus and the detected genotyping bands for each allele. KI, knock-in; L-HA, left homology arm; R-HA, right homology arm. (B) Genotyping PCR screening of floxed and deleted alleles, including floxed c-Maf (493 bp), Ayul-Cre (269 bp) and c-Maf deletion (993 bp). (C) HE staining of lens sections from control (c-Mafflff) and $c$-Maf $f^{f / f}$; Ayul-Cre mice at E14.5. The control mice were $c$-Mafflft. (D) Immunostaining for c-MAF in lens fiber cells of control $\left(c-M a f^{+/+}\right)$and $c-M a f^{f l / f}$; Ayul-Cre mice at E14.5. (E) Genotyping PCR signals for deletion confirmed that $c$-Maf knockout was successfully induced by tamoxifen injection. All scale bars $=100 \mu \mathrm{m}$. 
null embryos show aplasia of the lens at E18.5 [10]. Hematoxylin and eosin (HE) staining revealed an abnormal lens structure in the homozygous knockout mice, which is similar previously reported HE staining in $c$ Maf null mouse embryos (Fig. 1C). The IHC results showed that c-MAF expression was completely depleted in lens fiber cells (Fig. 1D). These results demonstrated that we successfully achieved the integration of the floxed allele into the $c$-Maf target locus.

Next, for the purpose of investigating c-MAF functions in adult mice, we mated $c$-Maf $f^{f l / t}$ and $C A G-C r e-$ $E R^{T M}$ transgenic mice [5]. Genotyping PCR confirmed that tamoxifen-inducible $c$-Maf knockout mice $\left(c-M a f^{\triangle T A M}\right)$ were successfully generated (Fig. 1E).

\section{c-Maf $\triangle T A M$ mice displayed abnormal lens structure}

All of the four $c-M a f^{\triangle T A M}$ mice, which were analyzed 7 months after tamoxifen injection, exhibited an abnor- mal lens appearance when compared with that of the control mice. The white dot on lenses represents opacification of the lenses (Fig. 2A). Next, to histologically examine abnormal appearances in the $c-M a f^{\triangle T A M}$ mice, we conducted HE staining and analyzed the lens structure. Red squares show an anterior pole, a posterior pole and a germinative zone in the lens (Fig. 2B). Around the lenses of the $c$-Maf ${ }^{\triangle T A M}$ mice, lens epithelial-like cells were present and produced multiple layers, which was different from the control mice in which lens epithelial cells were in a monolayer and existed from the anterior pole to the germinative zone. In the germinative zone of the $c-M a f^{\triangle T A M}$ mice, differentiation was not maintained, and migration did not occur either. Moreover, lens epithelial cells were greatly amplified in the anterior pole; it was possible that the amplification led to a multilayered structure in the germinative zone and posterior pole instead of differentiation.

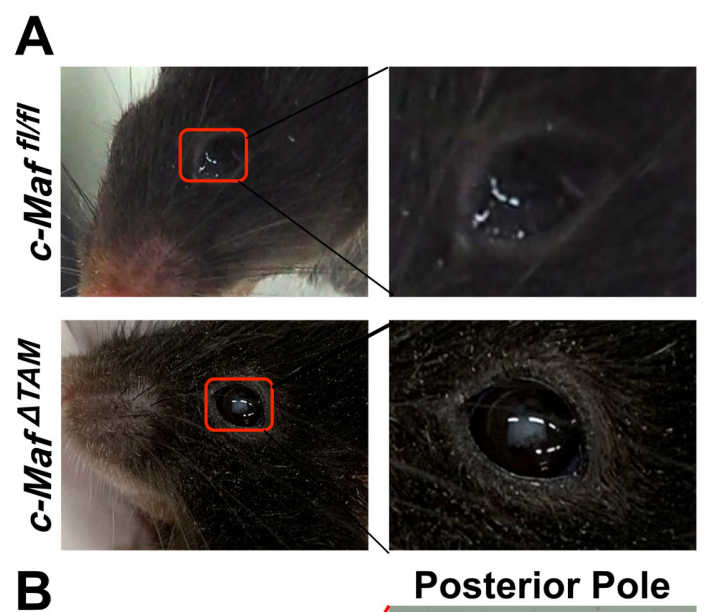

B

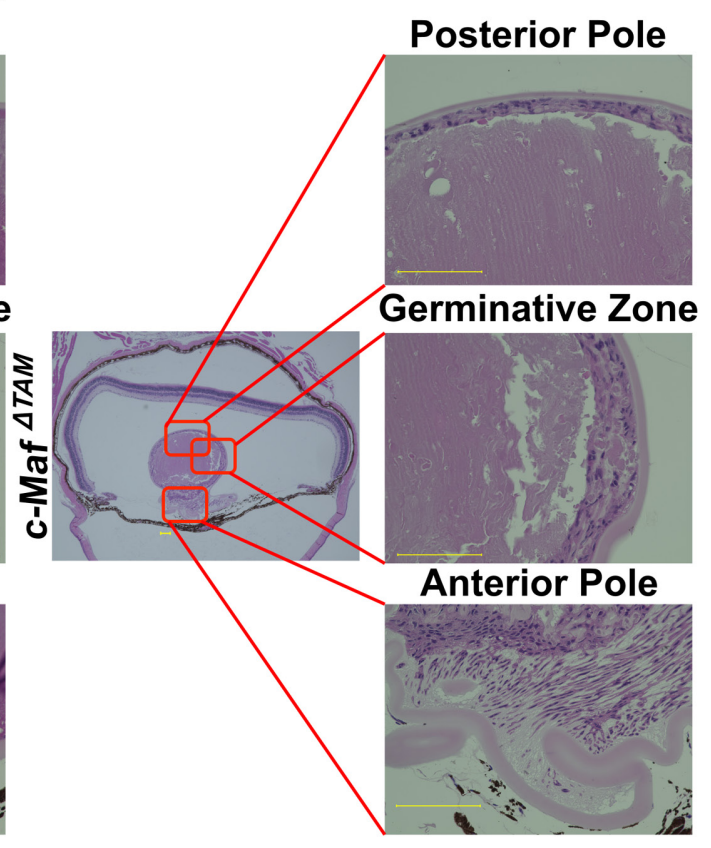

Fig. 2. $c$-Maf ${ }^{\triangle T A M}$ mice showed abnormal appearance of eye and lens structures. (A) Eyes of control and $c$-Maf $\triangle 4 A M$ mice. (B) HE staining for the anterior and posterior poles and germinative zone in the lenses of control and $c$-Maf ${ }^{\triangle T A M}$ mice. $\mathrm{n}=4$ each ( 1 male, 3 females). Scale bars $=100 \mu \mathrm{m}$. 
$c-M a f^{\triangle T A M}$ mice showed impaired differentiation and migration, and lens epithelial cells were observed in the posterior pole

We next performed IHC staining to identify which cells emerged on all sides of the lenses and what cells were present in the multiple layers around the lenses in the $c-M a f^{\triangle T A M}$ mice. Because E-cadherin is known as a marker of lens epithelial cells, we used antibodies against E-cadherin to detect lens epithelial cells. In the control mice, E-cadherin-positive lens epithelial cells (green) appeared to transition from the anterior pole to the germinative zone (Figs. 3B and C), and the c-MAF-positive lens fiber cells (pink) migrated from the germinative zone into the lens nuclear cells. (Fig. 3C). However, there were no epithelial cells at the posterior pole (Fig. 3D) of the control mice. In the $c-M a f^{\triangle T A M}$ mice, lens epithelial cells appeared in the three regions (Figs. 3B-D); in contrast, no lens fiber cells appeared in the germinative zone (Fig. 3B). In addition, lens epithelial cells (Ecadherin-positive) formed multiple layers around the lenses, which was unlike the single layers observed in the control mice (Figs. 3B-D). Moreover, the cells were arranged in a disordered row and displayed a disrupted structure in the anterior pole (Fig. 3B).

$c-M a f^{\triangle T A M}$ mice showed reduced crystallin genes expression

We next used qPCR to examine the expression of

A

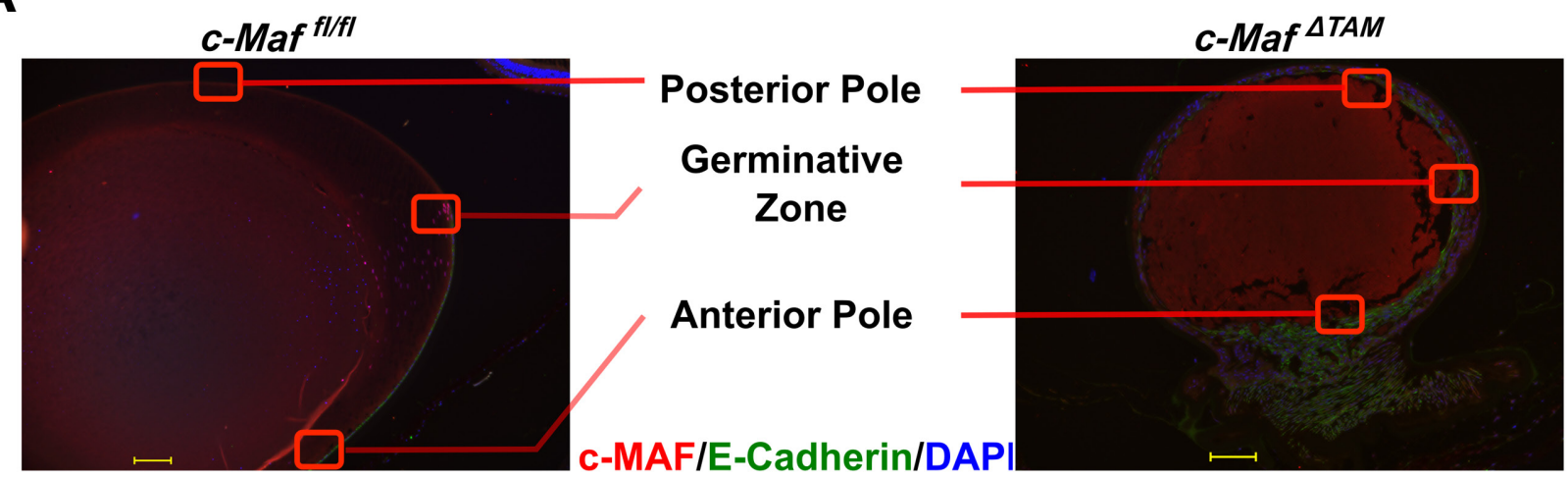

B

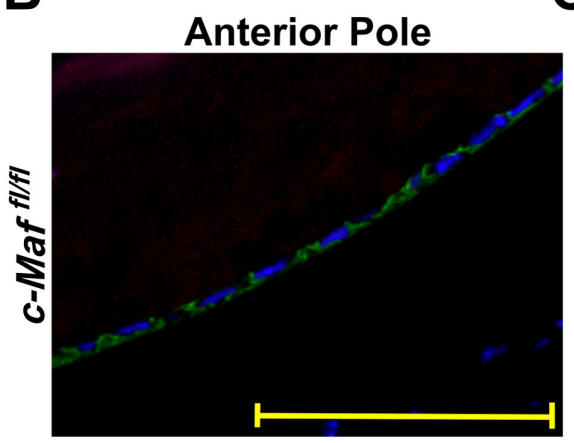

C

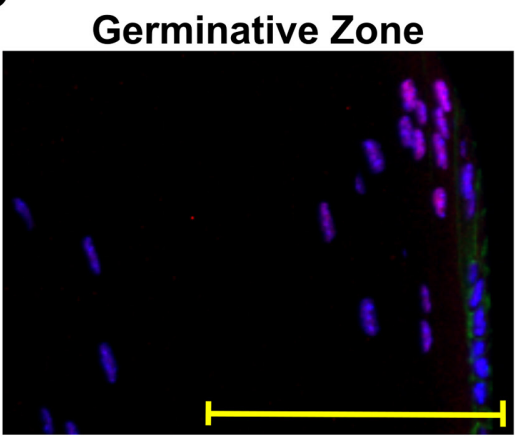

D
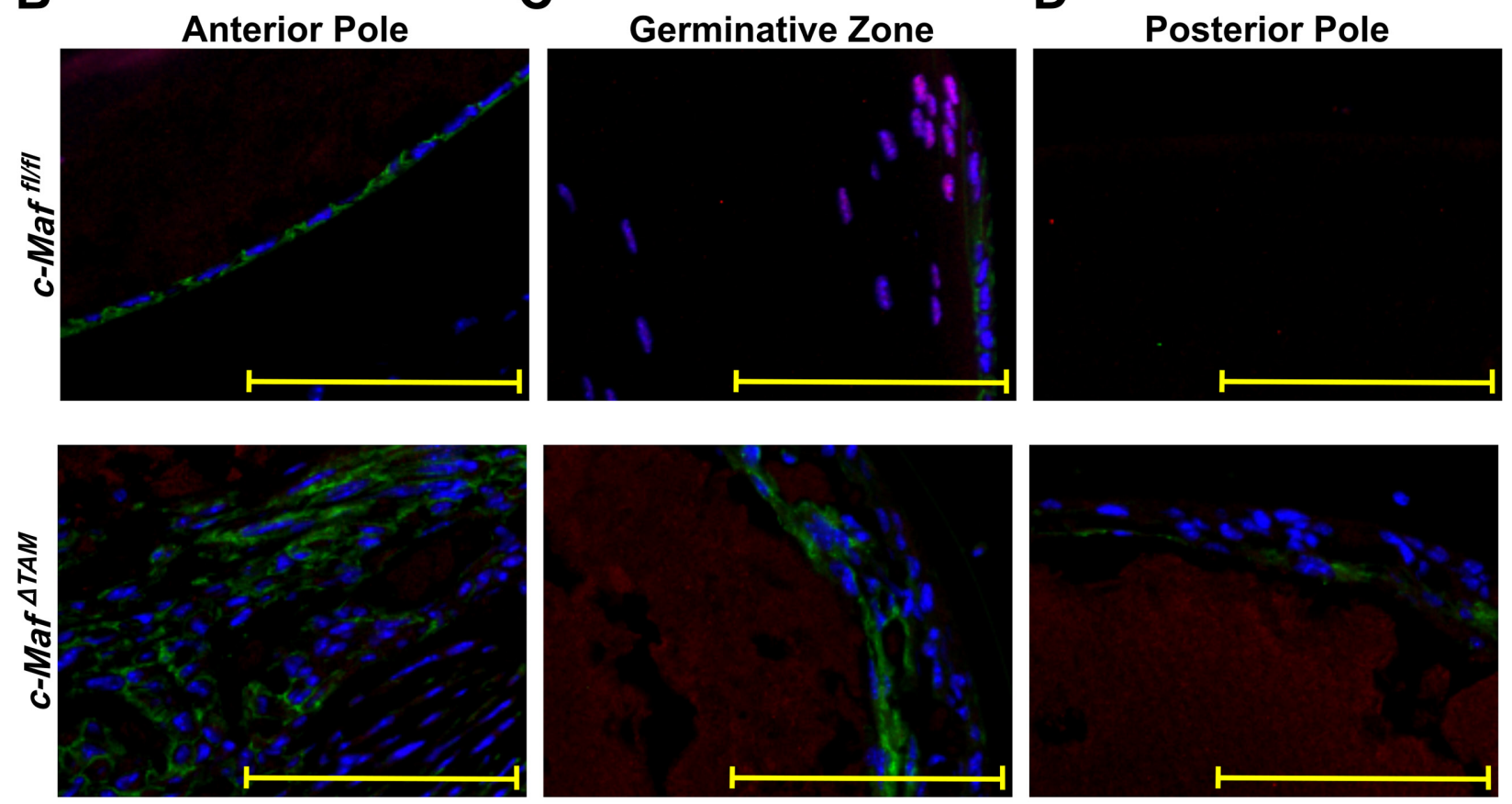

c-MAF/E-Cadherin/DAPI

Fig. 3. IHC revealed the expression of c-MAF and E-cadherin in the lenses of control and $c$-Maf ${ }^{4 T A M}$ mice. (A) Bright-field and IHC staining images of the lens. IHC staining for (B) germinative zone, (C) posterior and (D) anterior pole of the lens in the $c-M a f^{\Delta T A M}$ and control mice. IHC staining images are merged: E-Cadherin in green, c-MAF in red and DAPI in blue. $n=4$ each $(1$ male, 3 females). Scale bars $=100 \mu \mathrm{m}$. 


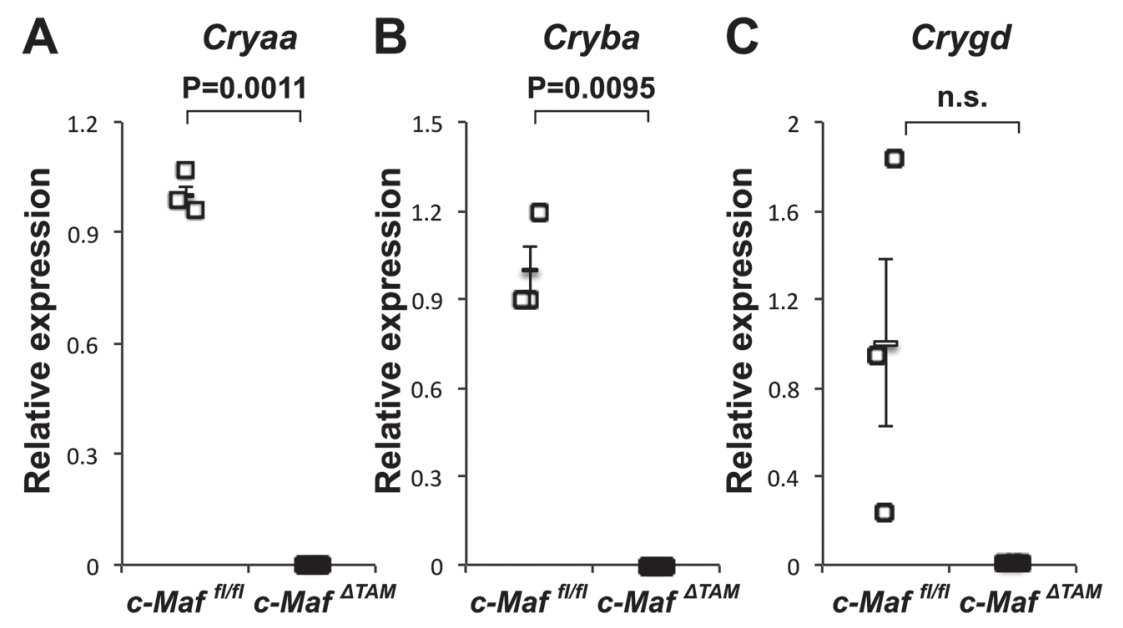

Fig. 4. c-Maf ${ }^{\triangle T A M}$ mice had decreased gene expression of Cryaa, Cryba and Crygd in the whole eye. Gene expression levels for Cryaa (A), Cryba (B), and Crygd (C) in the eyes of control and $c$-Maf ${ }^{\triangle T A M}$ mice 7 months after TAM injection. $\mathrm{n}=3$ each ( 1 male, 2 females). Scale bars $=100 \mu \mathrm{m}$.

crystallin genes, Cryaa, Cryba and Crygd, in the whole eye of $c-M a f^{\triangle T A M}$ and control mice. The results clearly indicated significant decreases in Cryaa ( $P$-value $=0.0011)$ and $C r y b a(P$-value $=0.0095)$ gene expression levels (Figs. 4A and B). There was no notable difference in the expression of Crygd $(P$-value $=0.164)$; however, there was almost no expression of Crygd in the $c-M a f^{\triangle T A M}$ mice (Fig. 4C). Taken together, $c$-Maf deletion leads to decreased gene expression of the lens crystallin genes in adult mice.

\section{Discussion}

The transcription factor c-MAF is associated with the differentiation of various cells and tissues, such as lens fiber cells $[10,11]$, subsets of T-lymphocytes [2, 6], hypertrophic chondrocytes during the development of endochondral bone [15, 19], and embryonic kidney and liver cells [8]. However, the physiological roles of c-MAF in adults have not been well studied because the homozygous deletion of $c-M a f$ in mice on a C57BL/6J background causes embryonic lethality [13]. Here, we generated $c$-Maf $f^{f l f t}$ mice and tamoxifen-inducible $c$-Maf conditional knockout mice $\left(c-M a f^{A T A M}\right)$. The $c-M a f^{f l f t}$; Ayul-Cre mice developed severe cataracts and lens anomalies, which are consistent with previous studies on $c$-Maf homozygous null embryos $[10,11]$. These results indicated that we successfully generated $c-M a f^{f l f t}$ and $c-M a f$ conditional knockout mice.

In previous studies, c-MAF was found to regulate Crystallin gene expression, and c-MAF deficiency induced aberrant lens structures. For example, c-MAF was shown to be essential for Cryaa expression and the in- duction of the differentiation of lens fibers [21]. c-MAF deficiency resulted in a noticeable decrease in $\gamma$-Crystallin gene expression and defective lens formation [11].c-Maf knockout embryos showed dysfunctional differentiation of lens epithelial cells into lens fiber cells, and they exhibited disrupted lens formation due to changes in Cryaa, Cryba and Crygd gene expression [10]. However, most previous studies focused on the fetal and postnatal stages of lens development and the associated abnormalities; one of the major reasons for this focus is the embryonic lethality observed in transgenic mice that was previously mentioned. In this study, we focused on the adult stage and defined c-MAF as the key factor in maintaining lens physiological structures.

First, Crystallin gene expression was significantly decreased in the $c-M a f^{\triangle T A M}$ mice. Previous studies using knockout mice showed the importance of crystallin gene expression for the maintenance of lens structures. For example, Cryaa is essential for the maintenance of lens transparency [3], Cryba is associated with age-related cataracts [22], and a Crygd deficiency in mice causes cataracts that are similar to human Coppock cataracts [24]. Our qPCR results showed a dramatic decrease in Cryaa, Cryba and Crygd expression in the c-Maf ${ }^{4 T A M}$ adult mice. Therefore, we demonstrate that c-MAF is related to Crystallin expression in adults. The reason why the expression levels of Crygd are different in each mouse is unknown, but according to Huang Y, et al. (Mol Vis. 2010 Mar 3; 16: 341-352.) [7], the expression level of Crygd is very low compared with $\alpha$ - and $\beta$-Crystallin in the rat lens. This low expression level might be the cause of the large variation.

Second, lens structures in $c-M a f^{\triangle T A M}$ mice were dra- 
matically different from control lenses. Previous studies showed that abnormal lens structures were caused by disorganization or by a lack of differentiation to produce lens fiber cells. For example, mice with a Lim 2 mutation show vacuolated lens structures and disorganized arrangement of fiber cells [25]; further, multiple layers of fiber-like cells in the central epithelium emerged in transgenic mice that overexpress TGF- $\beta$ in the lens [14]. Mice with a mutation in the Ercc2 gene demonstrate a delayed differentiation in the production of primary and secondary lens fiber cells [12]. Our HE and IHC staining results showed arrested differentiation and multiple layers of lens epithelial cells around the lenses due to a deficiency in c-MAF. Interestingly, there has been no evidence that the multiple layers are composed of lens epithelial cells. Since c-MAF can induce Crystallin expression, c-MAF acts as a differentiation switch in lens epithelial cells. Without c-MAF, lens epithelial cells cannot differentiate, so they do not stop proliferating. As a result, the epithelial cells in $c-M a f^{\triangle T A M}$ mice covered the whole lens and established multiple layers. Therefore, these results indicate that c-MAF is necessary for maintaining the polarity of the lens in adults.

Finally, opacification of lenses appeared in the $c-M a f^{\triangle T A M}$ mice. Opacification is one of the major phenotypes used to detect cataracts [17, 23, 27]. All three $c-M a f^{\triangle T A M}$ mice failed to maintain transparency in their lenses. In addition, our preliminary study demonstrated that the $c$-Maf ${ }^{\triangle T A M}$ mice 3 months after TAM treatment showed opacification and multiple layers of lens epithelial cells $(\mathrm{n}=1)$. Compared with the mice 3 months after TAM injection, the layers in the 7-month-old mice were spread more widely. Therefore, the results indicate that the stratification of the lens epithelial cells can be a timedependent change. Moreover, there are many papers showing that Cryaa is related to age-related cataracts. Considering these results and the observation that $c$-Maf ${ }^{\triangle T A M}$ mice clearly had cataracts, it is possible that c-MAF is related to age-related cataracts.

In conclusion, we applied CRISPR/Cas9 technology to generate $c$-Mafflft and $c$-Maf conditional knockout mice. Given that c-MAF is ubiquitously expressed in adults, c-MAF can play functional roles in various tissues. One example is that the $c-M a f^{\triangle T A M}$ mice had cataracts, and c-MAF was found to play an important role in the maintenance of lens physiological structures. Therefore, our $c$-Maf $f^{f l f l}$ mice could provide a useful model for a more detailed investigation of the physiological functions of c-MAF in each organ in adult mice.

\section{Conflict of Interest}

The authors declare no competing financial interests.

\section{Acknowledgments}

We thank Ms. Walla A. Basha for her help with immunostaining and Dr. Mitsuyasu Kato for providing an E-cadherin antibody for IHC staining. This work was supported by the Princess Takamatsu Cancer Research Fund and the JSPS KAKENHI Grant-in-Aid for Scientific Research (A) number 19H00966.

\section{References}

1. Blank, V., and Andrews, N.C. 1997. The Maf transcription factors: regulators of differentiation. Trends Biochem. Sci. 22: 437-441. [Medline] [CrossRef]

2. Blonska, M., Joo, D., Nurieva, R.I., Zhao, X., Chiao, P., Sun, S.C., Dong, C. and Lin, X. 2013. Activation of the transcription factor c-Maf in T cells is dependent on the CARMA1IKK $\beta$ signaling cascade. Sci. Signal. 6: ra110. [Medline] [CrossRef]

3. Brady, J.P., Garland, D., Duglas-Tabor, Y., Robison, W.G. Jr., Groome, A. and Wawrousek, E.F. 1997. Targeted disruption of the mouse alpha A-crystallin gene induces cataract and cytoplasmic inclusion bodies containing the small heat shock protein alpha B-crystallin. Proc. Natl. Acad. Sci. USA 94: 884-889. [Medline] [CrossRef]

4. Hansen, L., Eiberg, H. and Rosenberg, T. 2007. Novel MAF mutation in a family with congenital cataract-microcornea syndrome. Mol. Vis. 13: 2019-2022. [Medline]

5. Hayashi, S., and McMahon, A.P. 2002. Efficient recombination in diverse tissues by a tamoxifen-inducible form of Cre: a tool for temporally regulated gene activation/inactivation in the mouse. Dev. Biol. 244: 305-318. [Medline] [CrossRef]

6. Ho, I.C., Lo, D. and Glimcher, L.H. 1998. c-maf promotes T helper cell type 2 (Th2) and attenuates Th1 differentiation by both interleukin 4-dependent and -independent mechanisms. J. Exp. Med. 188: 1859-1866. [Medline] [CrossRef]

7. Huang, Y., and Xie, L. 2010. Expression of transcription factors and crystallin proteins during rat lens regeneration. Mol. Vis. 16: 341-352. [Medline]

8. Imaki, J., Tsuchiya, K., Mishima, T., Onodera, H., Kim, J.I., Yoshida, K., Ikeda, H. and Sakai, M. 2004. Developmental contribution of c-maf in the kidney: distribution and developmental study of c-maf mRNA in normal mice kidney and histological study of c-maf knockout mice kidney and liver. Biochem. Biophys. Res. Commun. 320: 1323-1327. [Medline] [CrossRef]

9. Jamieson, R.V., Perveen, R., Kerr, B., Carette, M., Yardley, J., Heon, E., Wirth, M.G., van Heyningen, V., Donnai, D., Munier, F. and Black, G.C. 2002. Domain disruption and mutation of the bZIP transcription factor, MAF, associated with cataract, ocular anterior segment dysgenesis and coloboma. Hum. Mol. Genet. 11: 33-42. [Medline] [CrossRef]

10. Kawauchi, S., Takahashi, S., Nakajima, O., Ogino, H., Morita, M., Nishizawa, M., Yasuda, K. and Yamamoto, M. 1999. Regulation of lens fiber cell differentiation by transcription factor c-Maf. J. Biol. Chem. 274: 19254-19260. [Medline] [CrossRef]

11. Kim, J.I., Li, T., Ho, I.C., Grusby, M.J. and Glimcher, L.H. 1999. Requirement for the c-Maf transcription factor in crystallin gene regulation and lens development. Proc. Natl. Acad. Sci. USA 96: 3781-3785. [Medline] [CrossRef] 
12. Kunze, S., Dalke, C., Fuchs, H., Klaften, M., Rössler, U., Hornhardt, S., Gomolka, M., Puk, O., Sabrautzki, S., Kulka, U., Hrabě de Angelis, M. and Graw, J. 2015. New mutation in the mouse $\mathrm{Xpd} / \mathrm{Ercc} 2$ gene leads to recessive cataracts. PLoS One 10: e0125304. [Medline] [CrossRef]

13. Kusakabe, M., Hasegawa, K., Hamada, M., Nakamura, M., Ohsumi, T., Suzuki, H., Tran, M.T., Kudo, T., Uchida, K., Ninomiya, H., Chiba, S. and Takahashi, S. 2011. c-Maf plays a crucial role for the definitive erythropoiesis that accompanies erythroblastic island formation in the fetal liver. Blood 118: 1374-1385. [Medline] [CrossRef]

14. Lovicu, F.J., Ang, S., Chorazyczewska, M. and McAvoy, J.W. 2004. Deregulation of lens epithelial cell proliferation and differentiation during the development of TGFbeta-induced anterior subcapsular cataract. Dev. Neurosci. 26: 446-455. [Medline] [CrossRef]

15. MacLean, H.E., Kim, J.I., Glimcher, M.J., Wang, J., Kronenberg, H.M. and Glimcher, L.H. 2003. Absence of transcription factor c-maf causes abnormal terminal differentiation of hypertrophic chondrocytes during endochondral bone development. Dev. Biol. 262: 51-63. [Medline] [CrossRef]

16. Metzger, D., and Chambon, P. 2001. Site- and time-specific gene targeting in the mouse. Methods 24: 71-80. [Medline] [CrossRef]

17. Morishita, H., Eguchi, S., Kimura, H., Sasaki, J., Sakamaki, Y., Robinson, M.L., Sasaki, T. and Mizushima, N. 2013. Deletion of autophagy-related 5 (Atg5) and Pik3c3 genes in the lens causes cataract independent of programmed organelle degradation. J. Biol. Chem. 288: 11436-11447. [Medline] [CrossRef]

18. Narumi, Y., Nishina, S., Tokimitsu, M., Aoki, Y., Kosaki, R., Wakui, K., Azuma, N., Murata, T., Takada, F., Fukushima, Y. and Kosho, T. 2014. Identification of a novel missense mutation of MAF in a Japanese family with congenital cataract by whole exome sequencing: a clinical report and review of literature. Am. J. Med. Genet. A. 164A: 1272-1276. [Medline] [CrossRef]

19. Nishikawa, K., Nakashima, T., Takeda, S., Isogai, M., Hamada, M., Kimura, A., Kodama, T., Yamaguchi, A., Owen, M.J.,
Takahashi, S. and Takayanagi, H. 2010. Maf promotes osteoblast differentiation in mice by mediating the age-related switch in mesenchymal cell differentiation. J. Clin. Invest. 120: 3455-3465. [Medline] [CrossRef]

20. Niwa, H., Araki, K., Kimura, S., Taniguchi, S., Wakasugi, S. and Yamamura, K. 1993. An efficient gene-trap method using poly A trap vectors and characterization of gene-trap events. $J$. Biochem. 113: 343-349. [Medline] [CrossRef]

21. Ogino, H., and Yasuda, K. 1998. Induction of lens differentiation by activation of a bZIP transcription factor, L-Maf. Science 280: 115-118. [Medline] [CrossRef]

22. Puk, O., Ahmad, N., Wagner, S., Hrabé de Angelis, M. and Graw, J. 2011. First mutation in the $\beta A 2$-crystallin encoding gene is associated with small lenses and age-related cataracts. Invest. Ophthalmol. Vis. Sci. 52: 2571-2576. [Medline] [CrossRef]

23. Shaham, O., Smith, A.N., Robinson, M.L., Taketo, M.M., Lang, R.A. and Ashery-Padan, R. 2009. Pax6 is essential for lens fiber cell differentiation. Development 136: 2567-2578. [Medline] [CrossRef]

24. Smith, R.S., Hawes, N.L., Chang, B., Roderick, T.H., Akeson, E.C., Heckenlively, J.R., Gong, X., Wang, X. and Davisson, M.T. 2000. Lop12, a mutation in mouse Crygd causing lens opacity similar to human Coppock cataract. Genomics 63: 314-320. [Medline] [CrossRef]

25. Steele, E.C. Jr., Kerscher, S., Lyon, M.F., Glenister, P.H., Favor, J., Wang, J. and Church, R.L. 1997. Identification of a mutation in the MP19 gene, Lim2, in the cataractous mouse mutant To3. Mol. Vis. 3: 5. [Medline]

26. Vanita, V., Singh, D., Robinson, P.N., Sperling, K. and Singh, J.R. 2006. A novel mutation in the DNA-binding domain of MAF at 16q23.1 associated with autosomal dominant "cerulean cataract" in an Indian family. Am. J. Med. Genet. A. 140: 558-566. [Medline] [CrossRef]

27. Zhao, H., Yang, T., Madakashira, B.P., Thiels, C.A., Bechtle, C.A., Garcia, C.M., Zhang, H., Yu, K., Ornitz, D.M., Beebe, D.C. and Robinson, M.L. 2008. Fibroblast growth factor receptor signaling is essential for lens fiber cell differentiation. Dev. Biol. 318: 276-288. [Medline] [CrossRef] 